\title{
Voltage-Gated Potassium Ion Channel Activation
}

National Cancer Institute

\section{Source}

National Cancer Institute. Voltage-Gated Potassium Ion Channel Activation. NCI

Thesaurus. Code C88550.

A process that involves detection of alteration to the voltage across a membrane, and results in opening of the channel to become selectively permeable to potassium ions. This interaction plays a crucial role in setting the potential of the resting membrane. 